[This is a post-peer-review, pre-copyedit version of an article published in Antonie van Leeuwenhoek. The final authenticated version is available online at: http://dx.doi.org/10.1007/s10482-018-01222-y]

\title{
Jiangella anatolica sp. nov. isolated from coastal lake soil
}

Hilal $\mathrm{Ay}^{1 *}$, Imen Nouioui ${ }^{2}$, Lorena Carro ${ }^{2 ¥}$, Hans-Peter Klenk ${ }^{2}$, Demet Cetin ${ }^{3}$, José M. Igual $^{4}$, Nevzat Sahin ${ }^{1}$, Kamil Isik ${ }^{5}$

${ }^{1}$ H. Ay, N. Sahin

Department of Molecular Biology and Genetics, Faculty of Science and Arts, Ondokuz Mayis University, Samsun, Turkey

*Corresponding author: hilal.ay@ omu.edu.tr, Tel: +90 362312 1919, Fax: +90 3624576081

${ }^{2}$ I. Nouioui, L. Carro, H.-P. Klenk

School of Natural and Environmental Sciences, Newcastle University, Ridley Building 2, Newcastle upon Tyne, NE1 7RU, UK

${ }^{¥}$ Present address: Dpto. de Microbiología y Genética. Universidad de Salamanca, Salamanca, 37007, Spain.

${ }^{3}$ D. Cetin

Science Teaching Programme, Gazi Faculty of Education, Gazi University, 06500, Ankara, Turkey

${ }^{4}$ J. M. Igual

Instituto de Recursos Naturales y Agrobiologia de Salamanca, Consejo Superior de Investigaciones Cientificas (IRNASA-CSIC), Salamanca, Spain

${ }^{5} \mathrm{~K}$. Isik

Department of Biology, Faculty of Science and Arts, Ondokuz Mayis University, Samsun, Turkey 


\section{Introduction}

The genus Jiangella was first proposed by Song et al. (2005) within the family Nocardioidaceae. However, Tang et al. (2011) established the family Jiangellaceae to accommodate the genera Jiangella (Song et al. 2005) and Haloactinopolyspora (Tang et al. 2011) mainly based on phylogenetic analysis of phylum Actinobacteria. Currently, the family Jiangellaceae includes the genera Jiangella, Haloactinopolyspora and the recently described Phytoactinopolyspora (Li et al. 2015). The type genus Jiangella encompasses aerobic, Gram-positive, filamentous actinomycetes with substrate mycelium fragmented into short and elongated rods. Members of the genus are characterized by the presence of LL-diaminopimelic acid in the cell-wall peptidoglycan and MK-9 $\left(\mathrm{H}_{4}\right)$ as the major menaquinone. None of the species described so far contain mycolic acids. The genus accommodates Jiangella gansuensis YIM $002^{\mathrm{T}}$ (Song et al. 2005), Jiangella alba YIM $61503^{\mathrm{T}}$ (Qin et al. 2009), Jiangella alkaliphila D8-87 ${ }^{\mathrm{T}}$ (Lee 2008), Jiangella muralis 15-Je-017 ${ }^{\mathrm{T}}$ (Kämpfer et al. 2011) and Jiangella mangrovi 3SM4-07 ${ }^{\mathrm{T}}$ (Suksaard et al. 2015) isolated from desert soil, surface sterilized stem of Maytenus austroyunnanensis, cave soil, cellar wall and mangrove soil, respectively.

Members of the phylum Actinobacteria are well-known for being prolific producers of most of the bioactive secondary metabolites (Goodfellow and Fiedler 2010). Thus, selective isolation of novel actinobacteria from unexplored habitats such as marine sediments (Carro et al. 2018) or lakes (Ay et al. 2017, 2018) holds considerable importance to discovery of novel bioactive compounds. In a continuation of our investigations on actinobacterial diversity in unexplored habitats a novel strain, GTF31 ${ }^{\mathrm{T}}$, was isolated from coastal soil sample of Gölcük Lake, a crater lake in southwest Anatolia (Turkey). Polyphasic characterization of strain GTF31 ${ }^{\mathrm{T}}$ showed that 
the strain is a novel species of the genus Jiangella, for which the name Jiangella anatolica sp nov is proposed.

\section{Materials and methods}

Isolation, maintenance and cultural conditions

Strain GTF31 ${ }^{\mathrm{T}}$ was obtained from coastal soil sample of Gölcük Lake, a freshwater lake, $\left(37^{\circ} 44^{\prime}\right.$ 03.90" $\mathrm{N} ; 30^{\circ} 29^{\prime} 39.24 "$ E) collected in September 2013. The strain was isolated on International Streptomyces Project Medium 2 (ISP 2 medium) (Shirling and Gottlieb 1966) supplemented with nystatin $(50 \mu \mathrm{g} / \mathrm{ml})$ and rifampicin $(5 \mu \mathrm{g} / \mathrm{ml})$ using standard dilution-plate technique. Briefly, the soil sample was suspended in $1 / 4$ strength Ringer's solution (Oxoid) and pretreated with wet heat at $60{ }^{\circ} \mathrm{C}$ for 20 minutes. Then, the diluted $\left(10^{-2}\right.$ and $\left.10^{-3}\right)$ suspensions were spread on ISP 2 medium. Inoculated plates were incubated at $28{ }^{\circ} \mathrm{C}$ for two weeks. The Jiangella isolate was purified and maintained on slopes of tryptone-yeast extract agar (DSM medium No. 680) at $4{ }^{\circ} \mathrm{C}$ and as glycerol stock solutions $(25 \% \mathrm{w} / \mathrm{v})$ at $-20^{\circ} \mathrm{C}$.

Cultural properties and phenotypic tests

Cultural and morphological characteristics were observed on ISP media $1-7$ (International Streptomyces Project, Shirling and Gottlieb (1966)), nutrient agar and tryptic soy agar (TSA; Difco) after incubation at $28{ }^{\circ} \mathrm{C}$ for 14 days. The ISCC-NBS Colour Charts Standard Samples No. 2106 (Kelly 1964) was used to determine the colours of aerial mycelium, substrate mycelium and diffusible pigments. Micromorphological characteristics were observed by scanning electron microscopy (JEOL JSM 6060) using cultures grown on glucose-yeast extract-malt extract (DSMZ Medium No. 65) at $28{ }^{\circ} \mathrm{C}$ for 14 days. The temperature $\left(4,10,20,28,37,50{ }^{\circ} \mathrm{C}\right)$ and $\mathrm{pH}$ range $(7$ -12 , at intervals of $1.0 \mathrm{pH}$ unit) were determined on ISP 2 medium (Shirling and Gottlieb 1966). Enzyme profile of the strain was established using the commercial kit API ZYM (BioMerieux) following the manufacturer's instructions. Carbon source utilization and chemical sensitivity 
properties were determined using GEN III Microplates in an Omnilog device (BIOLOG Inc., Hayward, CA, USA). The GEN III Microplates were inoculated with cells suspended in a viscous inoculation fluid (IF C) provided by manufacturer at $85-87 \%$ of transmittance for strain GTF $31^{\mathrm{T}}$ and the closely related type strains. The strains were studied in two independent technical replicates. Data were exported and analysed using opm package for R (Vaas et al. 2013; Vaas et al. 2012).

\section{Chemotaxonomy}

Biomass for chemotaxonomic analyses was obtained by cultivation in shake flasks at $28{ }^{\circ} \mathrm{C}$ for 14 days on a rotary shaker $(150 \mathrm{rpm})$ using glucose-yeast extract-malt extract (DSMZ Medium No. 65). Biomass was harvested by centrifugation at $9000 \mathrm{rpm}$, washed several times with sterile distilled water and then freeze-dried. Whole-cell amino acids and sugars were extracted following a standard protocol described by Lechevalier and Lechevalier (1970) and analysed by thin layer chromatography (TLC) according to Staneck and Roberts (1974). Polar lipids were extracted and analysed by two-dimensional TLC following Minnikin et al. (1984) using modifications of Kroppenstedt and Goodfellow (2006). Menaquinones were extracted from freeze-dried biomass and purified according to Collins (1985). The extracted menaquinones were analysed using a high performance liquid chromatography (HPLC; LC-10AS, Shimadzu 63 Co.) fitted with a reversephase C18 column $(150 \times 4.6 \mathrm{~mm}, 5 \mu \mathrm{m}$ particle size, RP-18-Lichrosorb column, Penomenex UK) at $270 \mathrm{~nm}$. Fatty acid methyl esters were extracted from fresh cultures grown on tryptic soy agar (TSA; Difco) at $28{ }^{\circ} \mathrm{C}$ for 10 days and analysed using Microbial Identification System (MIDI) Sherlock software version 6.1 and the library RTSBA6 according to the technical instructions provided by this system (Sasser 1990).

Phylogeny

Genomic DNA was extracted as described by Chun and Goodfellow (1995) and PCR amplification of $16 \mathrm{~S}$ rRNA gene achieved using the universal primers $27 \mathrm{~F}\left(5^{\prime}-\right.$ 
AGAGTTTGATC(AC)TGGCTCAG-3') and 1492R (5'-ACGG(CT)TACCTTGTTACGACTT3') (Weisburg et al. 1991). The purified PCR products were sequenced using an ABI PRISM 3730 $\mathrm{XL}$ automatic sequencer and an almost complete 16S rRNA gene sequence (1491 bp) was deposited in the GenBank data library and corresponding sequences of the type strains of the genus Jiangella were retrieved by using the EzBioCloud server (Yoon et al. 2017). A multiple sequence alignment was generated with MUSCLE program (Edgar 2004). Neighbour joining tree (Saitou and Nei 1987) was constructed in MEGA 6 (Tamura et al. 2013) using the model of Jukes and Cantor (Jukes and Cantor 1969). Phylogenetic analyses for maximum likelihood (ML) and maximum parsimony (MP) methods were conducted by the GGDC web server using the DSMZ phylogenomics pipeline (Meier-Kolthoff et al. 2014) adapted to single genes after pairwise sequence similarities were calculated according to Meier-Kolthoff et al. (2013a) via the GGDC web server (Meier-Kolthoff et al. 2013b) available at http://ggdc.dsmz.de/. ML tree was inferred from the alignment with RAxML (Stamatakis 2014) using rapid bootstrapping in conjunction with the autoMRE criterion (Pattengale et al. 2010) and MP tree was inferred from the alignment with TNT (Goloboff et al. 2008) using 1000 bootstraps in conjunction with tree-bisection-andreconnection branch swapping and ten random sequence additional replicates. The sequences were checked for compositional bias using the $\mathrm{X}^{2}$ test as implemented in PAUP* (Swofford 2002).

The genome of strain GTF31 ${ }^{\mathrm{T}}$ was sequenced using the Illumina HiSeq 2500 next generation sequencing platform with a 250-bp paired end protocol (MicrobesNG, Birmingham, United Kingdom) and the draft genome sequence was submitted to NCBI under the accession number POTW01000000. The genome sequence was annotated on Rapid Annotations Using Subsystems Technology (RAST) server (Aziz et al. 2008). A phylogenomic tree was built on PATRIC web server (https://patricbrc.org/) (Wattam et al. 2016) using FastTree algorithm (Price et al. 2010). The digital DNA-DNA hybridization $(\mathrm{dDDH})$ similarities between the draft genome sequence of strain GTF31 $1^{\mathrm{T}}$ and publicly available genome sequence data of its close phylogenetic neighbours 
were determined using formula 2 of the GGDC web server (Meier-Kolthoff et al. 2013b). In addition, the level of pairwise genome-based similarities was evaluated based on the average nucleotide identity (ANI) value determined by using online ANI calculator described by Yoon et al. (2017) available at https://www.ezbiocloud.net/tools/ani.

\section{Results and discussion}

Strain GTF31 ${ }^{\mathrm{T}}$ was found to grow well on ISP 1, ISP 2, ISP 3, ISP 6, nutrient agar and tryptic soy agar while moderate on ISP 5 and ISP7 media. The strain could not grow on ISP 4 medium and no diffusible pigment was observed on any media tested nor did produce melanin pigment. Substrate mycelium fragmented into elongated rods $(2.1 \mu \mathrm{m} \times 0.3 \mu \mathrm{m})$ while aerial mycelium and spore formation could not be observed during 14-day incubation period on any media tested (Fig 1). The strain was able to grow at temperature and $\mathrm{pH}$ range of $20-37{ }^{\circ} \mathrm{C}$ (optimum at $28{ }^{\circ} \mathrm{C}$ ) and $6-12$ (optimum at $\mathrm{pH} 7-8$ ), respectively, and in the presence of $1 \% \mathrm{NaCl}$. Strain GTF31 ${ }^{\mathrm{T}}$ could oxidise various compounds including acetic acid, acetoacetic acid, D-arabitol, D-cellobiose, dextrin, gelatin, $\beta$-gentiobiose, D-glucose, N-acetyl-D-glucosamine, $\beta$-methyl-D-glucoside, glycerol, L-lactic acid, D-maltose, D-mannitol, N-acetyl- $\beta$-D-mannosamine, pectin, D-salicin, sucrose, D-trehalose and turanose. The strain was able to respirate in presence of aztreonam, lincomycin, nalidixic acid, potassium tellurite, rifamycin SV, $1 \%$ sodium lactate and troleandomycin whereas it could not tolerate fusidic acid, guanidine hydrochloride, lithium chloride, minocycline, niaproof, sodium bromate and tetrazolium blue (Table 1). Allantoin and urea hydrolyses and nitrate reduction tests were positive while arbutin hydrolysis was negative. In addition, strain GTF31 ${ }^{\mathrm{T}}$ produced alkaline phosphatase, esterase (C4), esterase lipase (C8), lipase (C14), cystine arylamidase, trypsine, $\alpha$-chymotrypsin, acid phosphatase, naphtol-AS-BIphosphohydrolase, $\alpha$-galactosidase, $\beta$-galactosidase, $\beta$-glucuronidase, $\alpha$-glucosidase, $\beta$ glucosidase, $\mathrm{N}$-acetyl- $\beta$-glucosaminidase, $\alpha$-mannosidase, $\alpha$-fucosidase as shown by API ZYM (BioMerieux) test. 
Chemotaxonomic characteristics of strain $\mathrm{GTF} 31^{\mathrm{T}}$ were consistent with those of the genus Jiangella such as LL-diaminopimelic acid and glucose in whole cell hydrolysate. The strain was also found to contain mannose, rhamnose and ribose. Polar lipid profile was comprised of diphosphatidylglycerol, phosphatidylglycerol, phosphatidylinositol, four glycophospholipids and three unidentified phospholipids (Fig S1). The predominant menaquinone was determined to be MK-9 $\left(\mathrm{H}_{4}\right)$. The major cellular fatty acids were identified as anteiso- $\mathrm{C}_{15: 0}(28.7 \%)$, iso- $\mathrm{C}_{16: 0}$ $(16.9 \%)$ and anteiso- $\mathrm{C}_{17: 0}(16.4 \%)$ (Table S1).

An almost complete 16S rRNA gene (1491 bp) was obtained and analysed on EzBiocloud server and also on GGDC web server. Phylogenetic analyses of 16S rRNA gene sequences showed that strain GTF31 ${ }^{\mathrm{T}}$ is a member of the genus Jiangella. The strain was seen to be closely related to $J$. gansuensis DSM $44835^{\mathrm{T}}$, J. alba DSM $45237^{\mathrm{T}}$, J. muralis DSM $45357^{\mathrm{T}}$, J. mangrovi 3 SM4-07 ${ }^{\mathrm{T}}$ and J. alkaliphila DSM $45079^{\mathrm{T}}$ with $16 \mathrm{~S}$ rRNA gene sequence similarity of 99.4\%, 99.3\%, 99.2\%, $99.1 \%$ and $98.8 \%$, respectively. Strain GTF31 ${ }^{\mathrm{T}}$ was found to form a distinct branch at the periphery of Jiangella 16S rRNA gene trees constructed by both neighbour joining (Fig 2) and maximum likelihood (Fig S2) tree-making algorithms.

The draft genome sequence of strain GTF31 ${ }^{\mathrm{T}}$ was deposited on GenBank with accession number of POTW00000000 and annotated with RAST server. The genome is composed by 256 contigs with N50 value of 54,053. The numbers of coding sequences and RNAs are 6444 and 51, respectively. Total genome size is $7 \mathrm{Mb}$ and $\mathrm{G}+\mathrm{C}$ content is $72.5 \%$. In phylogenomic tree constructed on PATRIC web server, strain GTF $31^{\mathrm{T}}$ formed a distinct cluster with other members of the genus Jiangella (Fig 3). The calculated digital DDH values between strain GTF31 ${ }^{\mathrm{T}}$ and type species of J. gansuensis, J. alba, J. muralis and J. alkaliphila ranged from 24.4 to $34.4 \%$, values which are well below the proposed threshold of $70 \%$ for species delineation (Wayne et al. 1987). In addition to low DDH values, ANI values calculated on EzBioCloud web server (Table S2), which ranged between 81.0 - $87.9 \%$ (value below the threshold of 95-96\%) (Richter and 
Rosselló-Móra 2009), confirmed differentiation of strain GTF $31^{\mathrm{T}}$ from its phylogenetic neighbours.

On the basis of phylogenetic, chemotaxonomic and morphological analyses, strain GTF $31^{\mathrm{T}}$ showed characteristics of the genus Jiangella but differs from previously described species. Therefore, it is clear that strain GTF $31^{\mathrm{T}}$ represents a novel member of the genus Jiangella for which Jiangella anatolica sp nov. is proposed.

Description of Jiangella anatolica sp. nov.

Jiangella anatolica (a.na.to'li.ca. N. L. fem. adj. anatolica, referring the place from which the type strain was isolated).

Aerobic, Gram-positive actinomycete that forms mycelia fragmenting into elongated rods. Substrate mycelium is cream or pebble grey on ISP 1, ISP 2, ISP 3, ISP 5, ISP 6, ISP 7, nutrient agar and tryptic soy agar media. Shows good growth on ISP $1-3$, ISP 6 , nutrient agar and tryptic soy agar media while moderate on ISP 5 and ISP 7 media. Optimal growth occurred at $28{ }^{\circ} \mathrm{C}$ and pH 7 - 8. Tolerates aztreonam, lincomycin, nalidixic acid, potassium tellurite, rifamycin SV, 1\% sodium chloride, $1 \%$ sodium lactate and troleandomycin. Positive for allantoin and urea hydrolyses, nitrate reduction tests and production of acid phosphatase, alkaline phosphatase, $\alpha$ chymotrypsin, cystine arylamidase, esterase (C4), esterase lipase (C8), $\alpha$-fucosidase, $\alpha$ galactosidase, $\beta$-galactosidase, $\beta$-glucuronidase, $\alpha$-glucosidase, $\beta$-glucosidase, $N$-acetyl- $\beta$ glucosaminidase, lipase (C14), a-mannosidase, trypsine, naphtol-AS-BI-phosphohydrolase. Whole cell hydrolysate contains LL-DAP, glucose, mannose, rhamnose and ribose. The predominant menaquinone is $\mathrm{MK}-9\left(\mathrm{H}_{4}\right)$. The polar lipid profile consists of diphosphatidylglycerol, phosphatidylglycerol, phosphatidylinositol, glycophospholipids and unidentified phospholipids. Major fatty acids are anteiso- $\mathrm{C}_{15: 0}$, iso- $\mathrm{C}_{16: 0}$ and anteiso- $\mathrm{C}_{17: 0}$. The $\mathrm{G}+\mathrm{C}$ content of the type strain is $72.5 \%$ and the size of the genome is $7.0 \mathrm{Mb}$. 
The type strain GTF31 ${ }^{\mathrm{T}}\left(=\mathrm{DSM} 100984^{\mathrm{T}}=\right.$ CECT $\left.9378^{\mathrm{T}}\right)$ was isolated from coastal soil sample of Gölcük Lake, Isparta, Turkey. The GenBank/EMBL/DDBJ accession numbers of the $16 \mathrm{~S}$ rRNA gene sequence and whole genome sequence of strain GTF31 ${ }^{\mathrm{T}}$ are KU255546 and POTW01000000, respectively.

\section{Acknowledgements}

HA is grateful for the Scientific and Technological Research Council of Turkey (TÜBİTAK) for the PhD scholarship. IN and LC are grateful for Newcastle University for the postdoctoral fellowship.

\section{Author contributions}

HA, NS and KI designed the study. HA, IN and LC conducted physiological experiments. HPK, HA, IN, JMI designed chemotaxonomic analyses. DC carried out scanning electron microscopy analysis.

\section{Compliance with ethical standards}

\section{Conflict of interest}

The authors declare that they have no conflict of interest.

\section{Ethical approval}

This article does not contain any studies with human participants and/or animals performed by any of the authors. The formal consent is not required in this study.

\section{Supplementary material}

Table S1 Fatty acids composition of strain GTF31 ${ }^{\mathrm{T}}$, Jiangella gansuensis $\mathrm{JCM} 13367^{\mathrm{T}}$, J. alba JCM $16901^{\mathrm{T}}$, J. muralis JCM $17970^{\mathrm{T}}$

Table S2 Genome sequence comparison between strain GTF31 ${ }^{\mathrm{T}}$ and closely related type strains of the genus Jiangella. Genome accession numbers are given in brackets 
Fig. S1 Polar lipid profile of strain GTF31 ${ }^{\mathrm{T}}$. Key: DPG, diphosphatidylglycerol; PG, phosphatidylglycerol; PI, phosphatidylinositol; GPL1-4, unidentified glycophospholipids; PL13, unidentified phospholipid

Fig. S2 Maximum likelihood phylogenetic tree based on 16S rRNA gene sequences showing relationships between strain GTF31 ${ }^{\mathrm{T}}$ and closely related type strains. The tree was inferred under the GTR+GAMMA model. The numbers above the branches are support values when larger than $60 \%$ from maximum likelihood (left) and maximum parsimony (right) bootstrapping. The branches are scaled in terms of the expected number of substitutions per site 


\section{References}

Ay H, Nouioui I, del Carmen Montero-Calasanz M, Carro L, Klenk HP, Goodfellow M, Igual JM, Çetin D, Şahin N, Işık K. (2017) Actinomadura alkaliterrae sp. nov., isolated from an alkaline soil. Antonie van Leeuwenhoek 110(6):787-94

Ay H, Nouioui I, del Carmen Montero-Calasanz M, Klenk HP, Isik K, Cetin D, Sahin N (2018) Streptomyces sediminis sp. nov. isolated from crater lake sediment. Antonie van Leeuwenhoek, 111(4), 493-500

Aziz RK, Bartels D, Best AA, DeJongh M, Disz T, Edwards RA, Formsma K, Gerdes S, Glass EM, Kubal M, Meyer F (2008) The RAST Server: rapid annotations using subsystems technology BMC Genomics 9:75

Carro L, Veyisoglu A, Cetin D, Igual JM, Klenk HP, Trujillo ME, Sahin N (2018) A study of three bacteria isolated from marine sediment and description of Micromonospora globispora sp. nov. Syst Appl Microbiol https://doi.org/10.1016/j.syapm.2018.11.003

Chun J, Goodfellow M (1995) A phylogenetic analysis of the genus Nocardia with 16S rRNA gene sequences. Int J Syst Bacteriol 45:240-245

Collins MD (1985) 11 Analysis of isoprenoid quinones. In: Methods in microbiology, vol 18. Elsevier, pp 329-366

Edgar RC (2004) MUSCLE: multiple sequence alignment with high accuracy and high throughput Nucleic Acids Res 32:1792-1797

Goloboff PA, Farris JS, Nixon KC (2008) TNT, a free program for phylogenetic analysis Cladistics 24:774-786

Goodfellow M, Fiedler HP (2010) A guide to successful bioprospecting: informed by actinobacterial systematics. Antonie Leeuwenhoek 98(2):119-142

Jukes TH, Cantor CR (1969) Evolution of protein molecules Mammalian protein metabolism 3:132 
Kämpfer P, Schäfer J, Lodders N, Martin K (2011) Jiangella muralis sp. nov., from an indoor environment Int J Syst Evol Microbiol 61:128-131

Kelly K (1964) Color-name charts illustrated with centroid colors Inter-Society Color CouncilNational Bureau of Standards, Chicago

Kroppenstedt RM, Goodfellow M (2006) The family Thermomonosporaceae: Actinocorallia, Actinomadura, Spirillospora and Thermomonospora. In: The Prokaryotes. Springer, pp $682-724$

Lechevalier MP, Lechevalier H (1970) Chemical composition as a criterion in the classification of aerobic actinomycetes Int J Syst Evol Microbiol 20:435-443

Lee SD (2008) Jiangella alkaliphila sp. nov., an actinobacterium isolated from a cave Int J Syst Evol Microbiol 58:1176-1179

Li L et al. (2015) Phytoactinopolyspora endophytica gen. nov., sp. nov., a halotolerant filamentous actinomycete isolated from the roots of Glycyrrhiza uralensis Int J Syst Evol Microbiol 65:2671-2677

Meier-Kolthoff JP, Auch AF, Klenk H-P, Göker M (2013a) Genome sequence-based species delimitation with confidence intervals and improved distance functions BMC bioinform $14: 60$

Meier-Kolthoff JP, Göker M, Spröer C, Klenk H-P (2013b) When should a DDH experiment be mandatory in microbial taxonomy? Arch Microbiol 195:413-418

Meier-Kolthoff JP et al. (2014) Complete genome sequence of DSM 30083 T, the type strain (U5/41 T) of Escherichia coli, and a proposal for delineating subspecies in microbial taxonomy Stand Genomic Sci 9:2

Minnikin D, O'donnell A, Goodfellow M, Alderson G, Athalye M, Schaal A, Parlett J (1984) An integrated procedure for the extraction of bacterial isoprenoid quinones and polar lipids $\mathbf{J}$ Microbiol Methods 2:233-241

Pattengale ND, Alipour M, Bininda-Emonds OR, Moret BM, Stamatakis A (2010) How many bootstrap replicates are necessary? J Comput Biol 17:337-354 
Price MN, Dehal PS, Arkin AP (2010) FastTree 2-approximately maximum-likelihood trees for large alignments PloS one 5:e9490

Qin S, Zhao G-Z, Li J, Zhu W-Y, Xu L-H, Li W-J (2009) Jiangella alba sp. nov., an endophytic actinomycete isolated from the stem of Maytenus austroyunnanensis Int J Syst Evol Microbiol 59:2162-2165

Richter M, Rosselló-Móra R (2009) Shifting the genomic gold standard for the prokaryotic species definition. Proc Natl Acad Sci USA 106:19126-19131

Saitou N, Nei M (1987) The neighbor-joining method: a new method for reconstructing phylogenetic trees Mol Biol Evol 4:406-425

Sasser M (1990) Identification of bacteria through fatty acid analysis. In: Klement Z, Rudolph K, Sands D (eds) Methods in phytobacteriology. Akademiai Kiado, Budapest, pp 199-204

Shirling ET, Gottlieb D (1966) Methods for characterization of Streptomyces species Int J Syst Evol Microbiol 16:313-340

Song L, Li W-J, Wang Q-L, Chen G-Z, Zhang Y-S, Xu L-H (2005) Jiangella gansuensis gen. nov., sp. nov., a novel actinomycete from a desert soil in north-west China Int J Syst Evol Microbiol 55:881-884

Stamatakis A (2014) RAxML version 8: a tool for phylogenetic analysis and post-analysis of large phylogenies Bioinformatics 30:1312-1313

Staneck JL, Roberts GD (1974) Simplified approach to identification of aerobic actinomycetes by thin-layer chromatography Appl Microbiol 28:226-231

Suksaard P, Duangmal K, Srivibool R, Xie Q, Hong K, Pathom-Aree W (2015) Jiangella mangrovi sp. nov., isolated from mangrove soil Int J Syst Evol Microbiol 65:2569-2573

Swofford D (2002) PAUP* version 4.0 b10 Sinauer, Sunderland, MA

Tamura K, Stecher G, Peterson D, Filipski A, Kumar S (2013) MEGA6: molecular evolutionary genetics analysis version 6.0 Mol Biol Evol 30:2725-2729

Tang S-K, Zhi X-Y, Wang Y, Shi R, Lou K, Xu L-H, Li W-J (2011) Haloactinopolyspora alba gen. nov., sp. nov., a halophilic filamentous actinomycete isolated from a salt lake, with 
proposal of Jiangellaceae fam. nov. and Jiangellineae subord. nov Int J Syst Evol Microbiol 61:194-200

Vaas LA, Sikorski J, Hofner B, Fiebig A, Buddruhs N, Klenk H-P, Göker M (2013) opm: an R package for analysing OmniLog® phenotype microarray data Bioinformatics 29:18231824

Vaas LA, Sikorski J, Michael V, Göker M, Klenk H-P (2012) Visualization and curve-parameter estimation strategies for efficient exploration of phenotype microarray kinetics PloS one 7:e34846

Wattam AR et al. (2016) Improvements to PATRIC, the all-bacterial bioinformatics database and analysis resource center Nucleic Acids Res 45:D535-D542

Wayne L et al. (1987) Report of the ad hoc committee on reconciliation of approaches to bacterial systematics Int J Syst Evol Microbiol 37:463-464

Weisburg WG, Barns SM, Pelletier DA, Lane DJ (1991) 16S ribosomal DNA amplification for phylogenetic study J Bacteriol 173:697-703

Yoon S-H, Ha S-M, Kwon S, Lim J, Kim Y, Seo H, Chun J (2017) Introducing EzBioCloud: a taxonomically united database of $16 \mathrm{~S}$ rRNA gene sequences and whole-genome assemblies Int J Syst Evol Microbiol 67:1613-1617 
